\title{
Implementation Fidelity of a Complex Behavioral Intervention to Prevent Diabetes Mellitus in Two Safety Net Patient-Centered Medical Homes in New York City
}

Avni Gupta ( $\square$ avnigup07jan@gmail.com )

New York University https://orcid.org/0000-0003-4653-1272

Jiyuan Hu

New York University Grossman School of Medicine

Shengnan Huang

New York University Grossman School of Medicine

Laura Diaz

New York University Grossman School of Medicine

Radhika Gore

New York University Grossman School of Medicine

Natalie Levy

New York University Grossman School of Medicine

Michael Bergman

New York University Grossman School of Medicine

\section{Michael Tanner}

New York University Grossman School of Medicine

Scott E Sherman

New York University Grossman School of Medicine

Nadia Islam

New York University Grossman School of Medicine

Mark D Schwartz

New York University Grossman School of Medicine

\section{Research}

Keywords: Implementation fidelity, Process evaluation, Community health workers, Diabetes Prevention, Lifestyle intervention

Posted Date: August 3rd, 2021 
DOl: https://doi.org/10.21203/rs.3.rs-741692/v1

License: (c) (1) This work is licensed under a Creative Commons Attribution 4.0 International License. Read Full License 


\section{Abstract}

\section{Background}

It is critical to assess implementation fidelity for complex interventions to understand the reasons for their success or failure. However, few interventions systematically report implementation evaluation. Therefore, we conducted a concurrent process evaluation of CHORD (Community Health Outreach to Reduce Diabetes), a pragmatic, cluster-randomized, controlled trial to test the impact of a Community Health Workers (CHW) led, health coaching intervention on preventing incident type 2 Diabetes Mellitus (DM).

\section{Methods}

The study population included primary care (PC) patients with prediabetes at 2 safety-net hospitals - VA NY Harbor and Bellevue (BH). PC teams were randomized to receive the one-year intervention or usual care. Of the 559 patients in the intervention group to date, $79.4 \%$ completed an intake survey, constituting the analytic sample for fidelity assessment. The Conceptual Framework for Implementation Fidelity (CFIF) was applied to measure implementation fidelity and factors moderating fidelity of four core intervention components: patient goal setting, education topic coaching, PC visits, and referrals to address social determinants of health, using descriptive statistics and regression models.

\section{Results}

BH contributed $60.0 \%$ of the sample and VA contributed $40.0 \%$. Content adherence was high for three components with nearly $80.0 \%$ of patients setting $\geq 1$ goal, having $\geq 1 \mathrm{PC}$ visit and receiving coaching on $\geq 1$ education topic. Only $45.0 \%$ patients received $\geq 1$ referral. After adjusting for patient gender, language, race, ethnicity, and age, the study site moderated adherence to goal setting $(77.4 \% \mathrm{BH}$ vs. $87.7 \% \mathrm{VA})$, educational coaching (78.9\% BH vs. $88.3 \%$ VA), number of successful CHW-patient encounters (6 BH vs 4 VA) and percent of patients receiving all four components ( $41.1 \% \mathrm{BH}$ vs. $25.7 \% \mathrm{VA})$.

\section{Conclusions}

The CFIF enabled analysis of implementation fidelity of a complex, behavioral intervention. The fidelity of CHORD implementation varied across its four components and was moderated by site. Despite implementation in a research setting, interventions may not completely adhere to their core components, which can influence outcomes. Our study emphasizes the importance of examining implementation fidelity of interventions and of assessing moderating factors. Our study also empirically tested the CFIF using quantitative concurrent intervention fidelity evaluation.

Trial registration: The trial was registered with ClinicalTrials.gov on 12/30/2016 and the registration number is NCT03006666. The link to trial registration is:

https://clinicaltrials.gov/ct2/show/NCT03006666 


\section{Contributions To The Literature}

- Implementation of interventions in pragmatic trials can deviate from the protocol, but few studies report implementation fidelity, which is critical to translate them into practice.

- We evaluated the implementation of the CHORD trial intervention, a community health worker driven, complex behavioral program to prevent incident diabetes in at-risk patients and found variable implementation fidelity of its core components, which was moderated by implementation site.

- We empirically tested the Conceptual Framework of Implementation Fidelity and found that although the constructs provide a useful tool to measure fidelity and its moderators, further development is needed for a quantitative operationalization of moderators.

\section{Introduction}

Implementation fidelity, defined as the degree to which an intervention is implemented as planned, [1-3] is critical to inform our understanding of an intervention's outcomes [1,3-5] by avoiding the so called Type III error where a useful intervention could be rejected for showing a lack of impact when in reality the lack of impact was caused by suboptimal implementation fidelity [1]. In addition to clarifying causal mechanisms, assessment of implementation process can provide critical information to policy makers and evidence-based practitioners to guide future implementation of the intervention $[1-3,6]$. Examining implementation fidelity is particularly necessary for multicomponent complex interventions because of their higher likelihood of deviation from the protocol [6]. However, there are limited published studies of fidelity assessment of complex behavioral interventions $[6,7]$. A review of psychosocial interventions found that only $3.5 \%$ of the interventions adequately addressed fidelity.[3]

The CHORD trial (Community Health Outreach to Reduce Diabetes), launched in 2017, is a clusterrandomized pragmatic trial that is testing a Community Health Worker (CHW) driven intervention to promote healthy lifestyle changes and reduce the incidence of diabetes among a population of underserved patients with prediabetes in New York City safety-net hospital settings [8] There is evidence supporting the role of lifestyle interventions in preventing Type II diabetes mellitus (DM) among people with prediabetes and of the role of CHW's peer support in supporting positive behavioral and lifestyle transformations [9-11]. As behavioral transformations are difficult to achieve, primary care (PC) visits are often insufficient and peer support, a strategy that focuses on training individuals from within the community, such as $\mathrm{CHWs}$, to guide, educate, motivate, refer, track and support individuals to make and sustain behavior changes, can supplement PC visits to provide the required health coaching and social support for attaining behavior change goals [12]. CHWs are trained members of the public health workforce who are trusted members of the community and are familiar with local resources available for the community members $[8,13,14]$. They serve to bridge the cultural and health literacy gaps between communities and health care systems by providing culturally congruent and personalized peer-coaching, leveraging their shared lived experience with patients $[8,13,14]$. CHW driven interventions have demonstrated promising results in improving health knowledge, behaviors and outcomes, in particular for 
underserved communities $[13,15]$. The goal of the CHORD trial is to fill the gap in understanding how these strategies can be translated and implemented in the real world to foster positive behaviors in a population with prediabetes to prevent the onset of DM.

To balance the need for standardizing the intervention with responsivity to context $[13,16]$, the CHORD intervention was developed with defined core components and some optional components that allowed for varying intensities of implementation. This multicomponent, pragmatic, and community-based trial makes it a complex behavioral intervention with a high likelihood of deviation in the implementation of its protocol [17]. The purpose of this study was to conduct quantitative concurrent assessment of the implementation fidelity of the core components of CHORD intervention and to examine factors affecting implementation fidelity using the Conceptual Framework for Implementation Fidelity.[1, 5]

\section{Methods}

\section{Description of the Intervention}

The CHORD trial targets patients with prediabetes from PC clinics of the Manhattan campus of the VA NY Harbor Healthcare System (VA) and Bellevue Hospital Center (BH), of New York City's municipal hospital system [8]. We described details of the protocol previously, [7] and will briefly summarize the core elements here. Eleven PC teams at the VA and four PC teams at BH were randomized within each site into intervention and control arms. Eligible patients were identified from PC panels if they were 18-75 years of age with at least one glycosylated hemoglobin ( $\mathrm{HbA} 1 \mathrm{c})$ result in the prediabetic range $(5.7-6.4 \%)$ in the 5 years prior to the start of the intervention, after excluding patients with a prior diabetes diagnosis. The trial included patients who could speak English or Spanish to communicate with the bilingual CHWs. Each eligible patient in the intervention group was assigned to a CHWs based on language and neighborhood. Each CHW reached out to their assigned patients for a maximum of 10 times in attempt to enroll, after which the patient was dropped from further outreach. Once consented and enrolled, patients completed an intake survey.

The intervention included a 6-month intensive period followed by a 6-month maintenance period with monthly follow-up calls. The CHORD intervention was a complex intervention with four core components that were delivered by $\mathrm{CHWs}$ during in-person or telephonic encounters with patients. A minimum of 12 encounters, 6 in each of the two intervention periods was required.

As shown in the logic model in Fig. 1, the theory of change of the intervention was based on the delivery of the four core components - establishing personalized goals, educating, supporting PC visits and making appropriate referrals - by $\mathrm{CHWs}$ to affect behavioral lifestyle modifications and prevent diabetes onset among patients with prediabetes. The first intervention component required $\mathrm{CHWs}$ to establish individualized goals with each patient and complete a 6-item, Patient Activation Measure (PAM).[18] These goals were then translated into a health action plan (HAP), tailored to each patients' goals, PAM score, and preferences. Using the HAP, CHWs chose among 22 educational topics organized into 5 
modules (i.e., prediabetes 101, healthful eating, MyPlate,[19] and plate portions, physical activity, and stages of change) to conduct education session(s) with patients, and provided an educational packet with information about diabetes, exercise and nutrition to prevent diabetes. Patients were encouraged to have at least one $\mathrm{PC}$ appointment during the intervention period and $\mathrm{CHWs}$ called patients before and/or after $\mathrm{PC}$ visits to encourage them to discuss diabetes prevention with the PC clinician or to review their after-visit summaries regarding diabetes. CHWs could also meet the patients at the clinic during their PC visit. Finally, CHWs discussed and facilitated referrals to hospital, community-based programs as needed. In addition to these core activities, CHWs conducted regular check-in phone calls with their patients and could decide to offer other activities, such as home visits or group activities as appropriate. Postintervention, patients were contacted by a different staff member to complete a follow-up survey to assess their experience and satisfaction with the intervention, and changes they made in their diabetes prevention behaviors. To facilitate and standardize the implementation of the intervention, all CHWs received comprehensive training at the start and had ongoing feedback during weekly team meetings and case review sessions. These meetings allowed for troubleshooting of any issues and fostered exchange of experiences across $\mathrm{CHW}$ and the research team, creating an environment of co-learning for best addressing concerns and challenges in implementation.

To manage $\mathrm{CHW}$ caseload limits, patient enrollment at the two implementation sites in the CHORD trial was staggered across four, overlapping waves. Each intervention arm patient was eligible to receive the 12-month intervention. As the COVID-19 pandemic hit New York City in March 2020, the implementation of the CHORD study was substantially impacted and required adaptation of trial procedures. Due to a lack of comparability of process indicators before and since the pandemic, this paper was restricted to the pre-pandemic CHORD trial waves, which includes the first three waves at $\mathrm{BH}$ and two waves at VA. Data for these waves was collected over nearly 2 years from the start of the CHORD trial (December 2017) until October 2019. This study was approved by the NYU Langone Health (Protocol \#:16-00690) and the VA (Protocol \#:1609) Institutional Review Boards.

\section{Guiding Framework}

Data were collected in accordance with the modified version [5], of the Conceptual Framework for Implementation Fidelity (CFIF) originally proposed by Carroll et al, [1]. The key indicator of implementation fidelity according to CFIF is adherence defined as "whether a program service or intervention is being delivered as it was designed or written" [1]. The domain of adherence is conceptualized as coverage ("what proportion of target group participated in the intervention") [5], content ("was each of the intervention components implemented as planned") [5], and dosage which includes duration and frequency ("amount of an intervention received by participants" [1], or "were the intervention components implemented as often and for as long

as planned") [5]. In addition, the framework proposes to measure factors that moderate fidelity of implementation to investigate the reasons underlying any observed heterogeneity between different implementations of an intervention or deviations from the expected intervention which when combined with the adherence data, can provide a complete picture of fidelity. Context ("what factors at political, 
economical, organizational, and work group levels affected the implementation") [5] and participant responsiveness ("how were the participants engaged with the intervention services") were measured in this study to evaluate their effect on adherence.

Figure 2 shows the modified version of the CFIF along with the fidelity measures and moderators examined for the CHORD trial.

\section{Data Collection}

The data collection tools that were used to retrieve data for this study included standardized, Research Electronic Data Capture (REDCap) [20], forms completed by CHWs for each intervention patient to record their demographics, outreach and enrollment information, baseline intake information, and weekly logs about their encounters with each patient. Electronic Health Records from VA and BH provided other descriptive data on study patients and their healthcare utilization.

\section{Measures}

We report on the following four fidelity measures and two moderators. Table 1 describes the measures and data sources mapped to the framework constructs. 
Table 1

Study Measures and Their Data Sources Mapped to the Framework Constructs

$\begin{array}{lll}\begin{array}{l}\text { Framework } \\ \text { construct }\end{array} & \text { Reported measure from the CHORD trial } & \begin{array}{l}\text { Corresponding } \\ \text { CHORD trial core } \\ \text { component if } \\ \text { applicable }\end{array}\end{array}$

FIDELITY

Coverage

Percent of outreached patients who were enrolled

N/A

Outreach

form

Percent of enrolled patients who completed N/A

Intake form

intake

Percent of intake patients who completed

the first core component of establishing at

Core Component 1

Goal Setting

Form

least one goal or a Health Action Plan ${ }^{a}$

Content

adherence
Percent of intake patients who established

at least one goal or a Health Action Plan ${ }^{a}$

Percent of intake patients who received coaching on at least one education topic

Percent of intake patients who received coaching on all education modules

Percent of intake patients who had at least 1 PC visit

Percent of intake patients who received at least one referral

Core Component 4

Core Component 1

Core Component 2

Core Component 2

Core Component 3

Goal Setting Form

Encounter Form

Encounter Form

Electronic

Health

Record

Encounter and Referral

Forms

$\begin{array}{ll}\begin{array}{ll}\text { Percent of intake patients who received at } \\ \text { least one successful encounter }\end{array} & \text { N/A } \\ \begin{array}{ll}\text { Percent of intake patients who received all } & \text { Core Components } \\ \text { four core components in some capacity } & 1-4\end{array}\end{array}$

Median number of goals established

Dose-
frequency

Median number of goals completed
Encounter

Form

Goal Setting,

Encounter and Referral

Forms

Core Component 1

Goal Setting Form

Core Component 1
Goal Setting Form

a Percent of intake patients who established at least one goal or completed establishing a Health Action Plan was operationalized as a measure of two fidelity constructs - content adherence and coverage - because this component was the first component that patients were required to complete in order to proceed with other components of the intervention

${ }^{b}$ Duration of follow-up is the time from beginning of outreaching till the last successful encounter 


\begin{tabular}{|c|c|c|c|}
\hline $\begin{array}{l}\text { Framework } \\
\text { construct }\end{array}$ & Reported measure from the CHORD trial & $\begin{array}{l}\text { Corresponding } \\
\text { CHORD trial core } \\
\text { component if } \\
\text { applicable }\end{array}$ & Data Source \\
\hline & $\begin{array}{l}\text { Median number of education sessions } \\
\text { delivered }\end{array}$ & Core Component 2 & $\begin{array}{l}\text { Encounter } \\
\text { Form }\end{array}$ \\
\hline & $\begin{array}{l}\text { Median number of education modules } \\
\text { discussed }\end{array}$ & Core Component 2 & $\begin{array}{l}\text { Encounter } \\
\text { Form }\end{array}$ \\
\hline & Median number of PC visits & Core Component 3 & $\begin{array}{l}\text { Electronic } \\
\text { Health } \\
\text { Record }\end{array}$ \\
\hline & Median number of referrals & Core Component 4 & $\begin{array}{l}\text { Encounter } \\
\text { and Referral } \\
\text { Forms }\end{array}$ \\
\hline & Median number of successful encounters & $\mathrm{N} / \mathrm{A}$ & $\begin{array}{l}\text { Encounter } \\
\text { Form }\end{array}$ \\
\hline Dose- duration & Median duration (days) of follow-up time $\mathrm{b}^{\mathrm{b}}$ & $\mathrm{N} / \mathrm{A}$ & $\begin{array}{l}\text { Encounter } \\
\text { Form }\end{array}$ \\
\hline \multicolumn{4}{|c|}{ MODERATING FACTORS } \\
\hline $\begin{array}{l}\text { Participant } \\
\text { responsiveness }\end{array}$ & $\begin{array}{l}\text { Baseline Patient Activation Measure Score } \\
\text { ( }<\text { median vs. } \geq \text { median })\end{array}$ & $\mathrm{N} / \mathrm{A}$ & Intake Form \\
\hline Context & Clinical site (VA vs. BH) & $\mathrm{N} / \mathrm{A}$ & Study Form \\
\hline \multicolumn{4}{|c|}{$\begin{array}{l}\text { apercent of intake patients who established at least one goal or completed establishing a Health } \\
\text { Action Plan was operationalized as a measure of two fidelity constructs - content adherence and } \\
\text { coverage - because this component was the first component that patients were required to complete } \\
\text { in order to proceed with other components of the intervention }\end{array}$} \\
\hline
\end{tabular}

1. Coverage was measured as the percent of outreached patients who were enrolled, percent of enrolled patients who completed intake, and percent of intake patients who continued with completing the first core component of establishing at least one goal or a HAP, i.e. received some component of the CHORD intervention.

2. Content adherence of the four core components was measured as the percent of intake patients who established at least 1 goal or a HAP, received coaching on at least one education topic, received coaching on all education modules, had at least 1 PC visit, received at least one referral and who received all four core components in some capacity. In addition, we report the percentage of patients who had at least 1 successful encounter.

3. Dose - frequency of the four core components was measured as the median number of goals established and completed, median number of education sessions delivered and modules discussed, 
median number of PC visits, median number of referrals, and the median number of successful encounters.

Dose-duration was measured as the median duration of follow-up defined as the time from beginning of outreach until the last successful encounter

4. Participant responsiveness was measured as the baseline Patient Activation Measure (PAM) score since activation has been associated with engagement with behavioral interventions. We categorized the PAM score as less than or equal to median vs. above the median.

5. Context was measured as the two clinical sites - BH and VA.

\section{Analysis}

Our primary analysis focused on fidelity measures and, as a secondary analysis, we assessed how these fidelity measures differed by (or were moderated by) PAM score and by clinical site. Fidelity measures of coverage, content adherence and dose were reported using applicable descriptive statistics which included percentage for categorical variables, or median with their inter-quartile ranges (IQRs) for continuous or count variables. To evaluate how fidelity measures were moderated by PAM score and clinical site, unadjusted $p$-value from Chi-square or Mann-Whitney $U$ test and adjusted $p$-value from regression models that controlled for gender, language, race, ethnicity and age, were computed. For the regression model - the first set of models treated PAM score as the primary independent variable, and the second set of models treated clinical site as the primary independent variable. Each set of models included separate models for each fidelity measure as the dependent variable. Logistic regression models were used for measures of coverage and content adherence which were modelled as binary variables (received or not received the respective component of the intervention). Linear regression models were used for fidelity measures of dosage that were continuous, and negative binomial regression models were computed to model the fidelity measure of dosage that was a count variable.

Among patients who completed intake $(n=444)$, the denominator for our fidelity assessment, 32 patients (7.2\%) were missing the PAM score, and were excluded from the analysis examining moderation of fidelity measures by PAM score. Chi-square test or Mann-Whitney $U$ test, as appropriate, were used to compare two population groups - how eligible/outreached patients differed by their enrollment status and how enrolled patients differed by their intake completion status. These comparisons were conducted for demographic characteristics including gender, language, race, ethnicity, age at outreach (years) and PAM score. A successful encounter between a patient and a CHW was defined as an encounter (after the completion of the intake survey) where the $\mathrm{CHW}$ was able to speak with the patient either in-person or by phone, or when a letter or a text message was delivered (that is, when no evidence of failure to deliver was apparent). All quantitative analysis was conducted in the R statistical software environment,[21] and statistical significance was based on an alpha of $5 \%$.

\section{Results}


Results presented below have been categorized into A) assessment of implementation fidelity measures (primary analyses) and B) the impact of moderators on fidelity measures (secondary analyses).

\section{A) Fidelity Measures}

\section{Coverage}

A total of 1449 eligible patients were assigned to $\mathrm{CHWs}$ for outreach. After excluding 471 patients with incorrect/no contact information or who could not be reached after 10 phone calls, an additional 416 patients declined to participate or deferred and another 3 patients were found to be ineligible. This left 559 patients who enrolled in the intervention arm. Among enrolled patients, 444 (79.4\%) completed an intake survey and were eligible to receive the intervention and comprised the analytic sample for the fidelity assessment (Fig. 3). Among the 1449 patients eligible for enrollment, the 559 enrolled and 890 unenrolled patients differed in their primary language, race, ethnicity, implementation site and median age at outreach (Table 2). However, among those enrolled, patients completing intake vs. those not completing intake were not significantly different on any of the measured characteristics (Table 3). Among intake patients, 362 received the first core component of establishing a HAP. 
Table 2

Characteristics of Patients Who Were Determined Eligible for the Intervention Arm (and were Outreached by CHWs), by Their Enrollment Status

\begin{tabular}{|c|c|c|c|}
\hline & Eligible & Not enrolled & Enrolled \\
\hline & $(N=1449)$ & $(N=890)$ & $N=559$ \\
\hline Gender (Male) & $881(60.8 \%)$ & $539(60.6 \%)$ & $342(61.2 \%)$ \\
\hline Missing & $2(0.1 \%)$ & $1(0.1 \%)$ & $1(0.2 \%)$ \\
\hline \multicolumn{4}{|l|}{ Language } \\
\hline English & $1143(78.9 \%)$ & $722(81.1 \%)$ & $421(75.3 \%)$ \\
\hline Spanish & $262(18.1 \%)$ & $140(15.7 \%)$ & $122(21.8 \%)$ \\
\hline Other & $44(3.0 \%)$ & $28(3.1 \%)$ & $16(2.9 \%)$ \\
\hline \multicolumn{4}{|l|}{ Race* } \\
\hline Asian & $67(4.6 \%)$ & $45(5.1 \%)$ & $22(3.9 \%)$ \\
\hline Black & $377(26.0 \%)$ & $197(22.1 \%)$ & $180(32.2 \%)$ \\
\hline White & $269(18.6 \%)$ & $154(17.3 \%)$ & $115(20.6 \%)$ \\
\hline Other & $254(17.5 \%)$ & $134(15.1 \%)$ & $120(21.5 \%)$ \\
\hline Unknown & $482(33.3 \%)$ & $360(40.4 \%)$ & $122(21.8 \%)$ \\
\hline \multicolumn{4}{|l|}{ Ethnicity* } \\
\hline Hispanic or Latino & $432(29.8 \%)$ & $220(24.7 \%)$ & $212(37.9 \%)$ \\
\hline Not Hispanic or Latino & $1009(69.6 \%)$ & $664(74.6 \%)$ & $345(61.7 \%)$ \\
\hline Unknown & $8(0.6 \%)$ & $6(0.7 \%)$ & $2(0.4 \%)$ \\
\hline \multicolumn{4}{|l|}{ Hospital } \\
\hline Bellevue & $919(63.4 \%)$ & $591(66.4 \%)$ & $328(58.7 \%)$ \\
\hline VA & $530(36.6 \%)$ & $299(33.6 \%)$ & $231(41.3 \%)$ \\
\hline \multicolumn{4}{|l|}{ Age (yrs) at outreach } \\
\hline Median [Q1, Q3] & $58.3[47.9,66.0]$ & $57.9[47.2,65.4]$ & $58.6[49.4,67.1]$ \\
\hline Missing & $2(0.1 \%)$ & $1(0.1 \%)$ & $1(0.2 \%)$ \\
\hline \multicolumn{4}{|l|}{ Pam score区 } \\
\hline Median [Q1, Q3] & $18.0[18.0,21.0]$ & NA & $18.0[18.0,21.0]$ \\
\hline Missing & 1037 (71.6\%) & NA & 147 (26.3\%) \\
\hline
\end{tabular}




\begin{tabular}{|llll|}
\hline & Eligible & Not enrolled & Enrolled \\
\cline { 2 - 3 } & $(\mathrm{N}=1449)$ & $(\mathrm{N}=\mathbf{8 9 0})$ & $\mathrm{N}=559$ \\
\hline$\nabla$ Only for patients who completed intake; ${ }^{*} \mathrm{p}<0.001$ & \\
\hline
\end{tabular}


Table 3

Characteristics of Patients Who Were Enrolled in the Intervention Arm, by their Intake Completion Status

\begin{tabular}{|c|c|c|c|}
\hline & Enrolled & Intake completed & Intake not completed \\
\hline & $N=559$ & $N=444$ & $N=115$ \\
\hline Gender (Male) & $342(61.2 \%)$ & $264(59.5 \%)$ & $78(67.8 \%)$ \\
\hline Missing & $1(0.2 \%)$ & $0(0 \%)$ & $1(0.9 \%)$ \\
\hline \multicolumn{4}{|l|}{ Language } \\
\hline English & $421(75.3 \%)$ & $335(75.5 \%)$ & $86(74.8 \%)$ \\
\hline Spanish & $122(21.8 \%)$ & $97(21.8 \%)$ & $25(21.7 \%)$ \\
\hline Other & $16(2.9 \%)$ & $12(2.7 \%)$ & $4(3.5 \%)$ \\
\hline \multicolumn{4}{|l|}{ Race } \\
\hline Asian & $22(3.9 \%)$ & $18(4.1 \%)$ & $4(3.5 \%)$ \\
\hline Black & $180(32.2 \%)$ & $138(31.1 \%)$ & $42(36.5 \%)$ \\
\hline White & $115(20.6 \%)$ & $92(20.7 \%)$ & $23(20.0 \%)$ \\
\hline Other & $120(21.5 \%)$ & $96(21.6 \%)$ & $24(20.9 \%)$ \\
\hline Unknown & $122(21.8 \%)$ & $100(22.5 \%)$ & $22(19.1 \%)$ \\
\hline \multicolumn{4}{|l|}{ Ethnicity } \\
\hline Hispanic or Latino & $212(37.9 \%)$ & $174(39.2 \%)$ & $38(33.0 \%)$ \\
\hline Not Hispanic or Latino & $345(61.7 \%)$ & $269(60.6 \%)$ & $76(66.1 \%)$ \\
\hline Unknown & $2(0.4 \%)$ & $1(0.2 \%)$ & $1(0.9 \%)$ \\
\hline \multicolumn{4}{|l|}{ Hospital } \\
\hline Bellevue & $328(58.7 \%)$ & $265(59.7 \%)$ & $63(54.8 \%)$ \\
\hline VA & $231(41.3 \%)$ & $179(40.3 \%)$ & $52(45.2 \%)$ \\
\hline \multicolumn{4}{|l|}{ Age (years) at outreach } \\
\hline Median [Q1, Q3] & $58.6[49.4,67.1]$ & $58.7[49.2,67.2]$ & $58.2[50.2,66.5]$ \\
\hline Missing & $1(0.2 \%)$ & $0(0 \%)$ & $1(0.9 \%)$ \\
\hline \multicolumn{4}{|l|}{ Pam score】 } \\
\hline Median [Q1, Q3] & $18.0[18.0,21.0]$ & $18.0[18.0,21.0]$ & NA \\
\hline Missing & $147(26.3 \%)$ & $32(7.2 \%)$ & $115(100 \%)$ \\
\hline
\end{tabular}




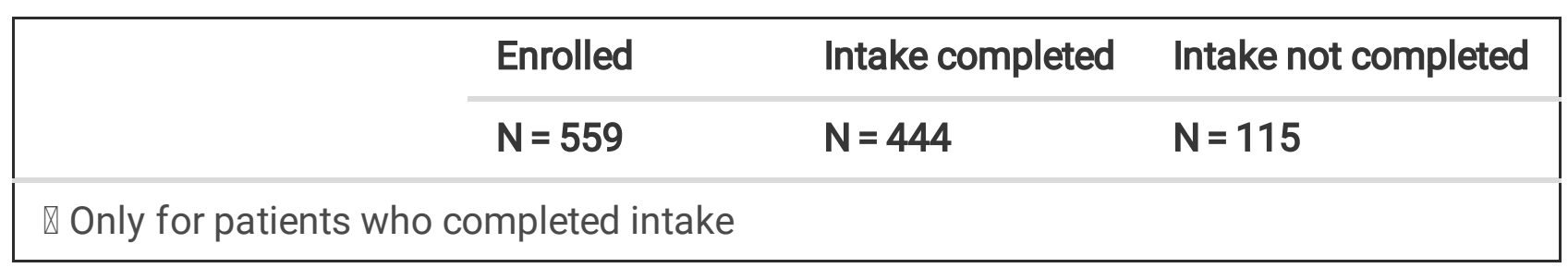

\section{Content adherence}

Nearly $80 \%$ of the patients completing an intake established at least one goal for their HAP, received coaching on at least one education topic and had at least one PC visit, indicating high content adherence to these three core components of the intervention. In addition, $40 \%$ received coaching on all education modules. Less than half (45.0\%) received a referral. Most of the referrals were for a healthcare visit, with the next most common reason for referrals being to facilitate access to healthy lifestyle, employment/workforce training, social security benefits, or mental health services. Nearly $80 \%$ of patients had at least one successful encounter with their CHW. Overall, $34.9 \%$ patients received all four core components in some capacity (Table 4). 
Table 4

Implementation Fidelity (Content Adherence) for Each Intervention Component

\begin{tabular}{|c|c|c|c|}
\hline Measure & $\begin{array}{l}\text { Relevant } \\
\text { core } \\
\text { component }\end{array}$ & $\begin{array}{l}\text { Number } \\
\text { completing the } \\
\text { component }\end{array}$ & $\begin{array}{l}\text { \% Completing } \\
\text { the component } \\
(\mathrm{n}=444)\end{array}$ \\
\hline $\begin{array}{l}\text { Percent of intake patients who established at } \\
\text { least one goal or a Health Action Plan }\end{array}$ & $\begin{array}{l}\text { Core } \\
\text { Component } \\
1\end{array}$ & 362 & $81.5 \%$ \\
\hline $\begin{array}{l}\text { Percent of intake patients who received } \\
\text { coaching on at least one education topic }\end{array}$ & $\begin{array}{l}\text { Core } \\
\text { Component } \\
2\end{array}$ & 367 & $82.7 \%$ \\
\hline $\begin{array}{l}\text { Percent of intake patients who received } \\
\text { coaching on all education modules }\end{array}$ & $\begin{array}{l}\text { Core } \\
\text { Component } \\
2\end{array}$ & 106 & $40.0 \%$ \\
\hline $\begin{array}{l}\text { Percent of intake patients who had at least } 1 \\
\text { PC visit }\end{array}$ & $\begin{array}{l}\text { Core } \\
\text { Component } \\
3\end{array}$ & 353 & $79.5 \%$ \\
\hline $\begin{array}{l}\text { Percent of intake patients who received at } \\
\text { least one referral }\end{array}$ & $\begin{array}{l}\text { Core } \\
\text { Component } \\
4\end{array}$ & 200 & $45.0 \%$ \\
\hline $\begin{array}{l}\text { Percent of intake patients who received at } \\
\text { least one successful encounter }{ }^{b}\end{array}$ & $\mathrm{~N} / \mathrm{A}$ & 354 & $79.7 \%$ \\
\hline $\begin{array}{l}\text { Percent of intake patients who received all } \\
\text { four core components in some capacity }\end{array}$ & $\begin{array}{l}\text { Core } \\
\text { Components } \\
1-4\end{array}$ & 155 & $34.9 \%$ \\
\hline \multicolumn{4}{|c|}{$\begin{array}{l}\text { a The number of patients who received coaching on at least one education topic were more than the } \\
\text { number of patients who established at least one goal (or a Health Action Plan) because although not } \\
\text { designed to, } 5 \text { patients received an educational coaching session before they established a goal (or a } \\
\text { Health Action Plan). }\end{array}$} \\
\hline \multicolumn{4}{|c|}{$\begin{array}{l}\text { b A successful encounter between a patient and a CHW was defined as an encounter (after the } \\
\text { completion of the intake survey) where the CHW was able to speak with the patient either in-person or } \\
\text { by phone, or when a letter or a text message was delivered (that is, when no evidence of failure to } \\
\text { deliver was apparent). The number of patients with a successful encounter is less than the number of } \\
\text { patients who completed at least one goal or the number of patients who received coaching on at least } \\
\text { one education topic because although not designed to, for some patients, after a patient completed } \\
\text { an intake, qoal establishment or education sessions happened without a successful encounter. }\end{array}$} \\
\hline
\end{tabular}

\section{Dose - Frequency and Duration}

Among the 362 patients who established a HAP, more than half established 3 goals (median 3; IQR: 2, 3), more than half completed at least 1 goal, and $25 \%$ completed 3 goals (median number of goals completed: 1 ; IQR: 0,3$)$. The median number of education sessions delivered was 18 (IQR: 8, 33) and the median number of education modules covered was 4 of 5 (IQR: 2, 4). Median number of referrals was 1 
(IQR: 1,3 ), with $25 \%$ receiving $\geq 3$. The median number of PC visits was 3 (IQR: 2,5 ). A total of 4,072 encounters were documented, of which $55.6 \%(n=2,155)$ were successful. The median number of successful encounters per patient was 5 (IQR: 3, 8) (Table 5). Among them, $40(9.9 \%)$ had the intended minimum number of successful encounters which was twelve. The median duration of follow-up time was 411 days [IQR: 341, 446]. Although the intervention was designed as a 6-month intensive period followed by a 6-month maintenance period, because of the real-world challenges of implementing a pragmatic community-based trial among vulnerable populations, the intervention phases did not adhere to the strict 6-month periods. Instead to accommodate variations in the responsiveness or needs of some patients, intensive contacts for some patients continued beyond 6 months and the overall intervention period extended over 12 months for some patients.

Table 5

Implementation Fidelity for Each Intervention Component

\begin{tabular}{|lll|}
\hline Measure & Relevant core component & Median [IQR] \\
\hline FREQUENCY & & \\
\hline Median number of goals established & Core Component 1 & $3[2,3]$ \\
\hline Median number of goals completed & Core Component 1 & $1[0,3]$ \\
\hline Median number of education sessions delivered & Core Component 2 & $18[8,33]$ \\
\hline Median number of education modules discussed & Core Component 2 & $4[2,4]$ \\
\hline Median number of PC visits & Core Component 3 & $3[2,5]$ \\
\hline Median number of referrals & Core Component 4 & $1[1,3]$ \\
\hline Median number of successful encounters & N/A & $5[3,8]$ \\
\hline DURATION & & \\
\hline Median duration (days) of follow-up time & N/A & $411[341,446]$ \\
\hline a Duration of follow-up is the time from beginning of outreaching till the last successful encounter & \\
\hline
\end{tabular}

\section{B) Impact of Moderators on Fidelity Measures Patient activation}

The median PAM score was 18.0 of a maximal score of 24 . As shown in Table 6, none of the fidelity measures was moderated by PAM score above versus below the median. 
Table 6

Moderation of Fidelity Measures by PAM Score Among Intervention Patients Completing the Intake Survey

\section{Fidelity Measure}

$\begin{array}{lllll}\begin{array}{l}\text { PAM } \\ \text { score } \\ \text { available } \\ \text { a }\end{array} & \begin{array}{l}\text { PAM } \\ \text { score }\end{array} & \begin{array}{l}\text { PAM } \\ \text { score }\end{array} & \begin{array}{l}\text { Unadjusted } \\ \text { P-value }\end{array} & \begin{array}{l}\text { Adjusted } \\ \text { P-value } \\ \text { b }\end{array} \\ \text { N=412 } & \begin{array}{l}\text { Median } \\ \text { Median }\end{array} & & \\ & \mathrm{N}=231 & \mathrm{~N}=181 & & \end{array}$

\section{COVERAGE}

Percent who completed the first core component of establishing at least one $80.8 \%$ $78.8 \%$ $83.4 \%$

0.289

0.259 goal or a Health Action Plan ${ }^{d}$

\section{CONTENT ADHERENCE}

Percent who established at least one $80.8 \%$

$78.8 \%$

$83.4 \%$

0.289

0.259

goal or a Health Action Plan ${ }^{d}$

Percent who received coaching on least one education topic

$81.8 \%$

$80.1 \%$

$84.0 \%$

0.375

0.411

Percent who received coaching on all education modules

$45.4 \%$

$44.2 \%$

$47.0 \%$

0.64

0.936

Percent who received at least one PC visit

Percent who received at least one referral

Percent who had at least one successful encounter

Percent who received all 4 core components in some capacity

$79.4 \%$

$78.8 \%$

$80.1 \%$

0.836

0.326

$43.4 \%$

$47.2 \%$

$38.7 \%$

0.103

0.305

$78.6 \%$

$78.4 \%$

$79.0 \%$

0.969

0.395

$33.3 \%$

$36.8 \%$

$28.7 \%$

0.105

0.611

\section{DOSE-FREQUENCY}

Median [IQR] number of goals established

$2[2,3]$

$3[2,3]$

$2[2,3]$

0.939

0.441

Median [IQR] number of goals completed $^{\mathrm{e}}$

Median [IQR] number of education sessions delivered

$1[0,2]$

$1[0,2]$

$1[0,2]$

0.760

0.553

Median [IQR] number of education modules covered

$17[7,31]$

17 [7,
$34]$

$17[8$,

0.634

0.943

Median [IQR] number of PC visits

$4[2,4]$

$4[2,4]$

$4[2,4]$

0.335

0.256

$3[2,5]$

$3[2,5]$

$3[2,5]$

0.117

0.180 


\begin{tabular}{|c|c|c|c|c|c|}
\hline Fidelity Measure & $\begin{array}{l}\text { PAM } \\
\text { score } \\
\text { available } \\
\text { a } \\
\mathrm{N}=412\end{array}$ & $\begin{array}{l}\text { PAM } \\
\text { score } \\
\leq \\
\text { Median } \\
\mathrm{N}=231\end{array}$ & $\begin{array}{l}\text { PAM } \\
\text { score } \\
\text { Median } \\
\text { N=181 }\end{array}$ & $\begin{array}{l}\text { Unadjusted } \\
\text { P-value }\end{array}$ & $\begin{array}{l}\text { Adjusted } \\
\text { P-value } \\
\text { b }\end{array}$ \\
\hline Median [IQR] number of referrals & $1[1,2.5]$ & $1[1,2]$ & $2[1,3]$ & 0.195 & 0.996 \\
\hline $\begin{array}{l}\text { Median [IQR] number of successful } \\
\text { encounters }\end{array}$ & $5[2,8]$ & $6[3,9]$ & $4[2,7]$ & 0.119 & 0.801 \\
\hline DOSE-DURATION & & & & & \\
\hline $\begin{array}{l}\text { Median [IQR] duration (days) of follow- } \\
\text { up time }\end{array}$ & $\begin{array}{l}405[334 \\
440]\end{array}$ & $\begin{array}{l}398 \\
{[335,} \\
439]\end{array}$ & $\begin{array}{l}408 \\
{[315} \\
440]\end{array}$ & 0.348 & 0.618 \\
\hline $\begin{array}{l}\text { All calculations are among subjects with } \\
\text { a Among patients who completed intake } \\
\text { b P values are adjusted for gender, langu } \\
\\
\text { c Two coverage measures - Percent of ol } \\
\text { patients who completed an intake - wer } \\
\text { our denominator (number of patients col } \\
\text { and Tables } 3 \text { and } 4 \text {. } \\
\text { d Percent of intake patients who establis } \\
\text { Action Plan was operationalized as a me } \\
\text { coverage - because this component wa } \\
\text { in order to proceed with other componen } \\
\text { e Calculated among subjects who establ }\end{array}$ & $\begin{array}{l}\text { n-missing } \\
\text { e, race, eth } \\
\text { eached pat } \\
\text { ot evaluate } \\
\text { leting intal } \\
\text { d at least o } \\
\text { ure of two } \\
\text { ef first com } \\
\text { of the inter } \\
\text { ed at least }\end{array}$ & $\begin{array}{l}\text { n't have a } \\
\text { sity and a } \\
\text { ts who } w \\
\text { for mode } \\
\text {. These n } \\
\text { goal or c } \\
\text { jelity cons } \\
\text { onent tha } \\
\text { ntion } \\
\text { ne goal }\end{array}$ & $\begin{array}{l}\text { PAM sco } \\
\text { e through } \\
\text { re enrolle } \\
\text { ation bec } \\
\text { easures } \\
\text { mpleted } \\
\text { ructs - } \\
\text { patients }\end{array}$ & $\begin{array}{l}\text { sure being rep } \\
\text { ecorded } \\
\text { nultivariate re } \\
\text { and Percent o } \\
\text { se they do no } \\
\text { e been report } \\
\text { tablishing a } \\
\text { tent adherenc } \\
\text { re required to }\end{array}$ & $\begin{array}{l}\text { rted } \\
\text { ressions } \\
\text { enrolled } \\
\text { make up } \\
\text { d in text } \\
\text { alth } \\
\text { and } \\
\text { omplete }\end{array}$ \\
\hline
\end{tabular}

\section{Context}

We found that the implementation of the CHORD trial was moderated by clinical site, a measure of context in our study. Nearly $60 \%$ in this analytic cohort were from BH and $40 \%$ from the VA. We found coverage and content adherence to be higher at VA than at $\mathrm{BH}$, except for the percent of patients who received coaching on all education modules and the percent of patients who received all four components in some capacity, which was higher at BH than at VA. Three content adherence measures, percent of patients who received at least one referral, at least one successful encounter and at least one $\mathrm{PC}$ visit were not different between the two sites. Three dose-frequency measures, number of PC visits (median value: 4 VA vs. $4 \mathrm{BH}$ ), number of education modules covered(median value: 4 VA vs. $4 \mathrm{BH}$ ), and the number of successful encounters (median value: 4.0 VA vs. $6.0 \mathrm{BH}$ ), differed between the two sites. (Table 7). 
Table 7

Moderation of Fidelity Measures by Clinical Site* Among Intervention Patients Completing the Intake Survey

\section{Fidelity Measure}

\section{Completed intake}

$(\mathrm{N}=444)$

\section{Bellevue Hospital}

$(\mathrm{N}=$

265)
VA Unadjusted

$(\mathrm{N}=\quad$ P-value 179)
Adjusted

P-value

a

COVERAGE ${ }^{b}$

Percent who completed the first core component (established at least one $81.5 \%$

$77.4 \%$

$87.7 \% \quad 0.008$

0.011

goal or a Health Action Plan) ${ }^{c}$

CONTENT ADHERENCE

Percent who established at least one

goal or a Health Action Plan ${ }^{c}$

Percent who received coaching

$82.7 \%$

$78.9 \%$

$88.3 \% \quad 0.015$

0.014

on least one education topic

Percent who received coaching

$40.0 \%$

$58.7 \%$

$47.5 \%<0.001$

0.003

on all education modules

Percent who received

$79.5 \%$

$82.3 \% \quad 75.4 \%$

0.102

0.134

at least one PC visit

Percent who received

$45.0 \%$

$47.9 \%$

$40.8 \%$

0.166

0.051

at least one referral

Percent who had at least

$79.7 \%$

$80.4 \%$

$78.8 \%$

0.770

0.422

one successful encounter

Percent who received all 4 core components in some capacity

$34.9 \%$

$41.1 \%$

$25.7 \%$

0.001

0.001

\section{DOSE-FREQUENCY}

Median [IQR] number

$3[2,3]$

$2[2,2]$

$2[2$,
$3]$

$<0.001$

0.128

of goals established

Median [IQR] number

$1[0,3]$

$0[0,2]$

$0[0$,
$2]$

0.921

0.731

of goals completed ${ }^{d}$

Median [IQR] number of

education sessions delivered

15 [7,
$31]$

20.5
$[9,34$ 


\begin{tabular}{|c|c|c|c|c|c|}
\hline Fidelity Measure & $\begin{array}{l}\text { Completed } \\
\text { intake } \\
(\mathrm{N}=444)\end{array}$ & $\begin{array}{l}\text { Bellevue } \\
\text { Hospital } \\
(\mathrm{N}= \\
265)\end{array}$ & $\begin{array}{l}\text { VA } \\
(\mathrm{N}= \\
179)\end{array}$ & $\begin{array}{l}\text { Unadjusted } \\
\text { P-value }\end{array}$ & $\begin{array}{l}\text { Adjusted } \\
\text { P-value } \\
\text { a }\end{array}$ \\
\hline $\begin{array}{l}\text { Median [IQR] number of } \\
\text { education modules covered }\end{array}$ & $4[2,4]$ & $4[2,4]$ & $\begin{array}{l}4[2, \\
4]\end{array}$ & 0.002 & 0.020 \\
\hline Median [IQR] number of PC visits & $3[2,5]$ & $3[2,5]$ & $\begin{array}{l}3[2 \\
5]\end{array}$ & 0.295 & $0.045^{\mathrm{e}}$ \\
\hline Median [IQR] number of referrals & $1[1,3]$ & $2[1,2]$ & $\begin{array}{l}1[1, \\
3]\end{array}$ & 0.179 & 0.242 \\
\hline $\begin{array}{l}\text { Median [IQR] number } \\
\text { of successful encounters }\end{array}$ & $5[3,8]$ & $6[3,10]$ & $\begin{array}{l}4[2 \\
7]\end{array}$ & 0.004 & $0.045^{\mathrm{e}}$ \\
\hline DOSE-DURATION & & & & & \\
\hline $\begin{array}{l}\text { Median [IQR] duration (days) } \\
\text { of follow-up time }\end{array}$ & $\begin{array}{l}411[341 \\
446]\end{array}$ & $\begin{array}{l}395 \\
{[313} \\
446]\end{array}$ & $\begin{array}{l}427 \\
{[375,} \\
446]\end{array}$ & 0.011 & 0.473 \\
\hline \multicolumn{6}{|c|}{$\begin{array}{l}\text { * All calculations are among subjects with non-missing data on the fidelity measure being reported } \\
\text { ap values are adjusted for gender, language, race, ethnicity and age through multivariate regressions } \\
\text { b Two coverage measures - Percent of outreached patients who were enrolled and Percent of enrolled } \\
\text { patients who completed an intake - were not evaluated for moderation because they do not make up } \\
\text { our denominator (number of patients completing intake). These measures have been reported in text } \\
\text { and Tables } 3 \text { and } 4 \text {. }\end{array}$} \\
\hline
\end{tabular}

\section{Discussion}

The theory of change of the CHORD intervention is that active, culturally congruent engagement of patients with prediabetes with trained $\mathrm{CHWs}$, through individualized goal setting, educational coaching, supplementation of PC visits and facilitated referrals, will support positive lifestyle changes and prevent the onset of diabetes. However, interventions will unlikely affect lifestyle change if they are not implemented with fidelity. This study conducted a concurrent process evaluation of the core components 
of the CHORD trial intervention, to examine the extent to which the study team successfully implemented each of the core components of the program (implementation fidelity). The study provided additional information for implementation efforts by examining the factors that influenced the extent of implementation (fidelity moderators).

Our analysis demonstrated moderate to high rates of implementation fidelity in this trial. We found that CHWs were able to complete an intake with nearly $80 \%$ of the patients enrolled in the intervention arm, and three of the four core components (goal setting, PC visit and education) were delivered to nearly $80 \%$ of the patients. While the component of facilitating a referral was delivered to only $45 \%$ of the patients, a quarter received 3 or more referrals. While coverage and content adherence were moderate to high for the four components, we found high variability in the dosage of these core components. As compared to a minimum of 6 required encounters per patient in the intensive and maintenance phase respectively, the median number of successful encounters in the intensive and maintenance phases in total was only 5(IQR: 3,8$)$. In addition, nearly $66 \%$ completed the intensive phase of the intervention, with an average follow-up time of more than a year. Given the planned duration of intervention, which was 1 year, the observed average follow-up time is a measure of implementation success.

In a complex intervention, there is potential for deviations from the planned program.[1] Lifestyle interventions, such as the one being tested by the CHORD trial, are inherently complex because they involve multiple dimensions, have diverse interacting components and target several organizational levels. This makes their success highly dependent on a multitude of real-world variables.[1, 2] These real world variables can precipitate deviation of the 'intervention as designed' from the 'intervention as delivered'.[22] Using multiple regression models we assessed the fidelity of implementation by two among the five moderating factors described in the modified CFIF,[1,5] a contextual factor (the site of implementation, $\mathrm{BH}$ vs. VA) and a measure of patient activation (PAM score). While the implementation site moderated several measures of content adherence and dosage, patient activation was not associated with fidelity.

Our study used PAM score to measure participant responsiveness. In light of evidence which shows that patient activation-"the skills and confidence that equip patients to become actively engaged in their health care" contributes to positive health outcomes,[23] null findings in our process evaluation were unexpected. However, these findings might be explained by emerging evidence indicating that interventions tailored to a patient's level of activation can build skills and confidence, thereby increasing patient activation.[23] The components of the CHORD trial were tailored to the patient activation measure at the time of intake, which might have affected their activation and engagement throughout the intervention. Therefore, a comparison of the quality of implementation by baseline PAM score might not have revealed any differences. It is also important to note that PAM score at intake might not be directly associated with the perception and view of patients about the relevance of an intervention, which has been suggested to directly moderate implementation fidelity by impacting the engagement of patients.[1] Hence, PAM score at intake might be limited in its representation of the concept of 'participant responsiveness' as defined in the CFIF, and of the concept echoed in Rogers' diffusion of innovations

Page 22/29 
which posits that "the uptake of a new intervention depends on its acceptance by those receiving it".[24] A mid or post intervention PAM measure or a qualitative assessment of patients' engagement could have altered implementation fidelity of the CHORD trial.

Two other moderators of implementation fidelity than those measured in our study have been suggested in the CFIF -'comprehensiveness of policy description' and 'strategies to facilitate intervention'[1, 25] Detailed specific descriptions of interventions and simple interventions are more likely to be implemented with high fidelity than ones that are vague.[1] In addition, support strategies such as the "provision of manuals, guidelines, training, and monitoring and feedback for those delivering the intervention", may moderate fidelity by optimizing and standardizing the intervention delivery, particularly critical for complex interventions.[1] Although we did not measure these moderators quantitatively - our description of the CHORD intervention can provide a guide to program administrators interested in replicating the intervention, by informing the approaches that were taken to facilitate the optimal delivery of the core components of the CHORD trial.

\section{Study limitations}

First, one of the inherent features of pragmatic trials which can pose challenges in accurately measuring implementation is that the intervention usually does not start or end on fixed days. Because CHWs maintain ongoing and continuing contacts with their patients, some of the interventions, such as referrals, were delivered outside of the intervention period. For the purpose of this analysis, these interventions were considered a part of the implementation if their information was available in the standard study data forms completed by CHWs. The CHORD protocol was developed to provide referrals during the intensive phase, but if needed, CHWs made some referrals even during the maintenance phase. All these interventions were considered part of CHORD implementation. Second, fidelity measures can be intervention specific. Therefore, the measures used in this study might not directly translate to other complex interventions. However, in the absence of detailed guidance about appropriate indicators for fidelity measures and moderators, this study adds to our knowledge base on the diversity of indicators that future evaluations can apply. Third, one important aspect of implementation is how well the participants engage with the intervention. In our study, while we measured the delivery of core components from the perspective of $\mathrm{CHWs}$, we could not assess the extent to which delivered interventions were received by patients. Fidelity is influenced by both, the people delivering as well as those receiving an intervention. Three dimensions of fidelity have been conceptualized: 1) Fidelity delivery is concerned with delivering an intervention as per protocol; 2) Fidelity receipt refers to participants' understanding of the intervention and their capacity to apply the information or skills; 3)Fidelity enactment reflects the extent to which participants can apply the delivered information or skills in actual situations.[6] Our analysis was not able to parse out these three dimensions.

\section{Study strengths and contributions to implementation science}


Fidelity assessment is key to understanding the reasons for the success or failure of interventions. However, very few studies systematically document and report implementation processes of their intervention programs.[3, 5$]$ The $\mathrm{CHORD}$ protocol built in collection of data regarding several fidelity measures and moderating factors that can impact fidelity. This allowed us to conduct concurrent rather than retrospective process evaluation. Concurrent process evaluations are important, as they can capture implementation experiences in real time and ensure that the theory behind the intervention is accounted for during evaluation.[6] In addition to reporting on concurrent process evaluation of a pragmatic complex trial, the study responds to the general calls for conducting quantitative evaluations of fidelity in intervention studies. Use of real time data reported by the key implementors, the CHWs adds to the validity of our analysis.

Another major contribution of our study is in empirically testing the CFIF. The framework provided a useful tool for conceptualizing and organizing measures of fidelity and their moderators. These findings, when evaluated with the data on CHORD's outcomes, can help address questions about the relative importance of the measures of implementation fidelity as described in the framework. These evaluations can also provide information on the predictive validity of fidelity measures. Our process evaluation also highlights the limitations of the CFIF for selecting measures of potential moderators. To standardize quantitative fidelity assessments, the field will benefit from further guidance about "how-to" measure and apply factors that can moderate implementation fidelity.

\section{Conclusion}

Our concurrent quantitative process evaluation of a complex pragmatic trial, using the Conceptual Framework for Implementation Fidelity, found moderate-to-high adherence to the core components of the trial, as well as moderation of several fidelity measures by implementation site. This study adds to the growing knowledge base on the evaluation of implementation fidelity of trials, which is important to understand "how" and "why" an intervention fails or succeeds. For complex behavioral interventions, such as the one evaluated in this study, fidelity assessment becomes further critical because of the high potential of deviation from the planned protocol. This empirical test of the framework found it to be a useful tool for organizing and measuring fidelity measures as well as their moderators. However, our process evaluation also highlights the current lack of guidance on quantitative indicators of the proposed moderators.

\section{Declarations}

Ethics approval and consent to participate: This study was approved by the NYU Langone Health (Protocol \#:16-00690) and the VA (Protocol \#:1609) Institutional Review Boards.

Consent for publication: Not applicable 
Availability of data and materials: The datasets used and/or analyzed during the current study are available from the corresponding author on reasonable request.

Competing interests: The authors declare that they have no competing interests

Funding: This study has undergone peer-review by and is supported by the National Institutes of Health National Institute of Diabetes and Digestive and Kidney Diseases under award number R18DK110740. The content is solely the responsibility of the authors and does not necessarily represent the official views of the National Institutes of Health. The funding agency has not influenced the design of this study or the writing of this manuscript and will not influence the study's implementation, data collection and analysis, and the writing of future manuscripts.

Authors' contributions: AG conceptualized the paper, wrote the first draft and finalized the subsequent revisions along with data interpretation and data presentation. $\mathrm{JH}$ led data management, data analyses and data presentation along with reviewing multiple versions of the manuscript to offer critical edits and comments. SH performed data management, data analyses and reviewed multiple versions of the manuscript to offer critical edits and comments. LD managed project implementation, performed data management and reviewed multiple versions of the manuscript to offer critical edits and comments. RG was involved with data interpretation, data presentation and also offered critical edits and comments to the manuscript. $\mathrm{NI}$ and MS conceptualized the trial and the manuscript along with providing key inputs to the content and data for the paper by reviewing multiple versions of the manuscript to offer critical edits and comments.

Acknowledgements: Presented in part at Society of General Internal Medicine (SGIM) 2021 conference and Academy Health ARM 2021 conference.

Authors' information (optional): Not applicable

\section{References}

1. Carroll C, et al. A conceptual framework for implementation fidelity. Implementation science. 2007;2(1):40.

2. Moore GF, et al. Process evaluation of complex interventions: Medical Research Council guidance. BMJ: British Medical Journal. 2015;350:h1258.

3. Breitenstein SM, et al. Implementation fidelity in community-based interventions. Res Nurs Health. 2010;33(2):164-73.

4. Damschroder LJ, et al. Fostering implementation of health services research findings into practice: a consolidated framework for advancing implementation science. Implementation Science. 2009;4(1):50.

5. Hasson H. Systematic evaluation of implementation fidelity of complex interventions in health and social care. Implementation Science. 2010;5(1):67. 
6. Ginsburg LR, et al. Examining fidelity in the INFORM trial: a complex team-based behavioral intervention. Implementation Science. 2020;15(1):78.

7. Dusenbury $L$, et al. A review of research on fidelity of implementation: implications for drug abuse prevention in school settings. Health Educ Res. 2003;18(2):237-56.

8. Islam N, et al. Protocol for the CHORD project (community health outreach to reduce diabetes): a cluster-randomized community health worker trial to prevent diabetes. BMC Public Health. 2018;18(1):1-11.

9. Absetz P, et al. Type 2 diabetes prevention in the "real world": one-year results of the GOAL Implementation Trial. Diabetes Care. 2007;30(10):2465-70.

10. Tseng $E$, et al. Survey of primary care providers' knowledge of screening for, diagnosing and managing prediabetes. J Gen Intern Med. 2017;32(11):1172-8.

11. Yarnall KS, et al. Primary care: is there enough time for prevention? American journal of public health. 2003;93(4):635-41.

12. Doull M, et al., Peer support strategies for improving the health and well-being of individuals with chronic diseases. The Cochrane Database of Systematic Reviews, 2017. 2017(6).

13. Agency for Healthcare Research. Outcomes of Community Health Worker Interventions. Agency for Healthcare Research and Quality: Rockville, MD; 2009.

14. Gore R, et al. Integrating Community Health Workers into Safety-Net Primary Care for Diabetes Prevention: Qualitative Analysis of Clinicians' Perspectives. J Gen Intern Med. 2020;35(4):1199-210.

15. Spencer MS, et al. Effectiveness of a community health worker intervention among African American and Latino adults with type 2 diabetes: a randomized controlled trial. American journal of public health. 2011;101(12):2253-60.

16. Haynes A, et al. Figuring out fidelity: a worked example of the methods used to identify, critique and revise the essential elements of a contextualised intervention in health policy agencies. Implementation Science. 2016;11(1):23.

17. Mihalic SF, Fagan AA, Argamaso S. Implementing the LifeSkills Training drug prevention program: factors related to implementation fidelity. Implementation Science. 2008;3(1):5.

18. Insignia Health. Patient Activation Measure. 6/29/2021]; Available from: https://www.insigniahealth.com/products/pam-survey.

19. US Department of Agriculture. MyPlate. [cited 2021; Available from: https://www.myplate.gov/.

20. Harris PA, et al. Research electronic data capture (REDCap)-A metadata-driven methodology and workflow process for providing translational research informatics support. J Biomed Inform. 2009;42(2):377-81.

21. Team RC, R: A language and environment for statistical computing. 2013.

22. Nelson MC, et al. A Procedure for Assessing Intervention Fidelity in Experiments Testing Educational and Behavioral Interventions. J Behav Health Serv Res. 2012;39(4):374-96. 
23. Hibbard JH, Greene J. What The Evidence Shows About Patient Activation: Better Health Outcomes And Care Experiences; Fewer Data On Costs. Health Aff. 2013;32(2):207-14.

24. Rogers EM. Diffusion of innovations. Simon and Schuster; 2010.

25. Hasson $\mathrm{H}$, Blomberg S, Dunér A. Fidelity and moderating factors in complex interventions: a case study of a continuum of care program for frail elderly people in health and social care. Implementation Science. 2012;7(1):23.

\section{Figures}

\section{Core input}

-Encounters between trained CHWs and patients with prediabetes range glycemia

\section{Process} core components to patients (Establish personalized goals, educate, support before or after PC

-CHWs deliver
core componen
patients (Establ
personalized go
educate, support
before or after
visit and make
appropriate
referrals)

\section{- CHWs deliv
core compo
patients (Es
personalized
educate, supp
before or af
visit and ma
appropriate
referrals) \\ -CHWs de
core comp
patients (
personalized
educate, s
before or
visit and
appropria
referrals)}

\section{Outcomes}

$\square$ Lifestyle modifications

-Weight change $\square$ Reduction in the incidence of diabetes

\section{Figure 1}

Logic Model and the Theory of Change of the CHORD Intervention 
Potential Moderators of implementation fidelity:

Participant responsiveness

Context

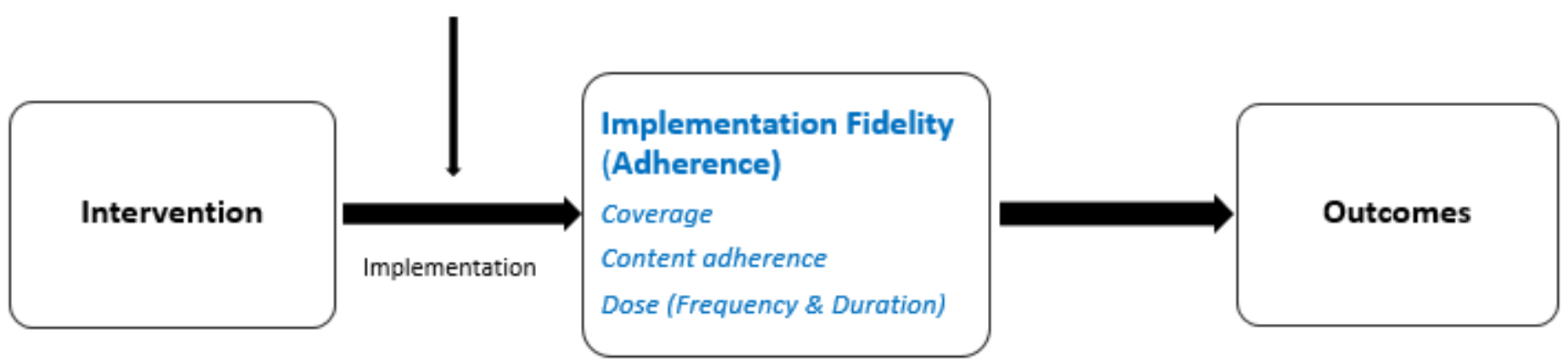

\section{Figure 2}

The modified version of the Conceptual Framework for Implementation Fidelity that guided fidelity assessment of the CHORD intervention (adapted from Carroll et al. and Hasson et al.) 


\section{CONSORT Flow Diagram}

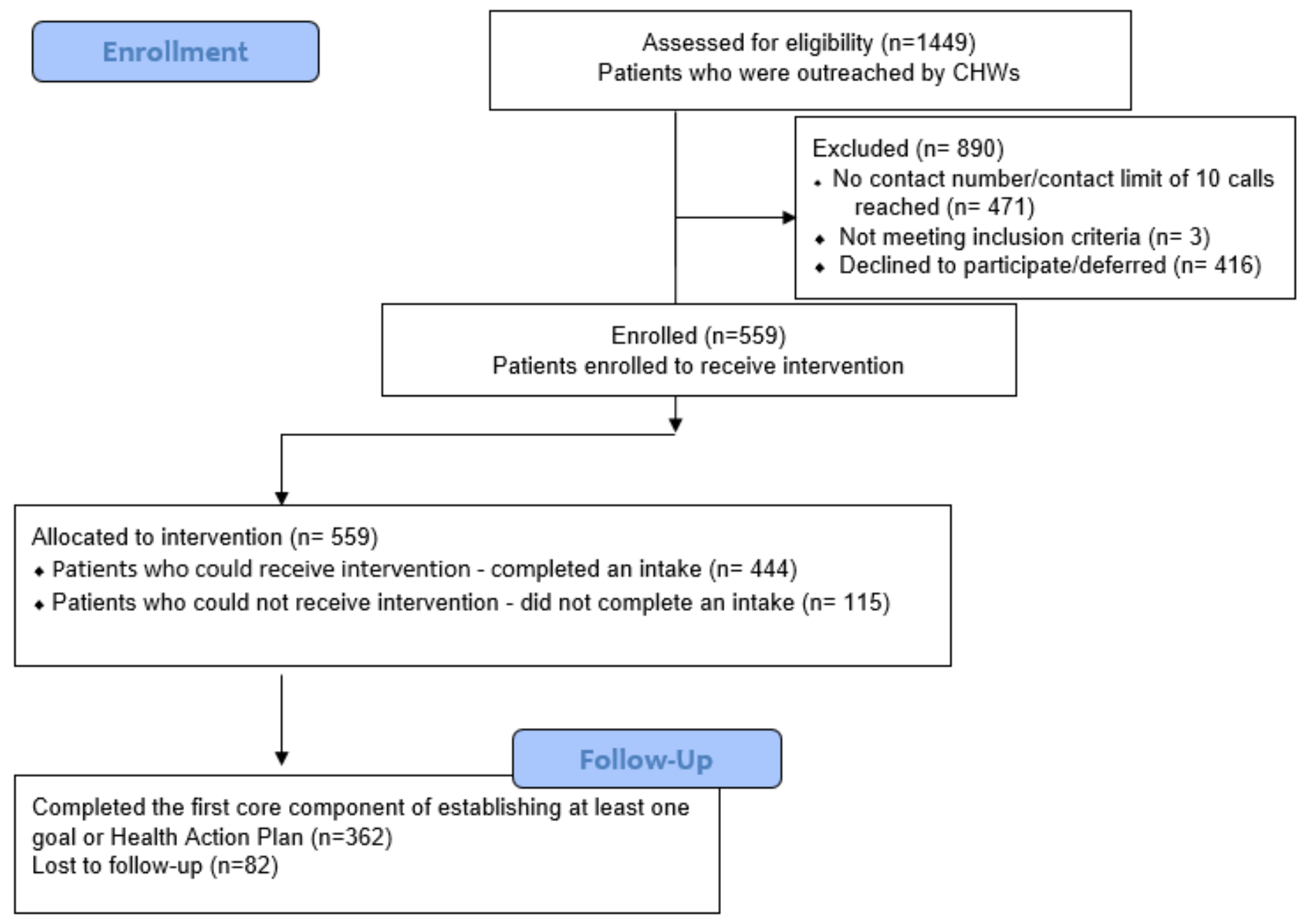

Figure 3

CONSORT flow diagram for the intervention arm 